\title{
Standardized Phenotyping: Advantages to Horticulture-Introduction to the Workshop
}

\author{
Nahla V. Bassil ${ }^{1}$ \\ National Clonal Germplasm Repository, USDA-ARS, 33447 Peoria Road, Corvallis, OR 97333-2521 \\ Gayle M. Volk \\ National Center for Genetic Resources Preservation, USDA-ARS, 1111 S. Mason Street, \\ Fort Collins, CO 80521
}

New and emerging DNA sequencing technologies have recently accelerated the pace of gene discovery. Phenotypes are the expression of genotypes and reveal gene function. As such, phenotypes provide the crucial link between sequence variation and biological function. As a result of the relative ease in obtaining vast quantities of genomic data, phenotypic information is not keeping pace with the explosion in available genomic information. The lack of reliable and available phenotypic data may limit the use of statistical methods to identify the associations between phenotypic and genotypic (or genomic) data, thus hindering our understanding of the genetic basis of biological processes. Sequence data reveal high levels of genomic syntenies that suggest conservation of gene order, content, and function among phylogenetically related groups. Although it is possible to assign putative gene function based on reported function in other plants and, in some instances, other organisms, inferences should be validated by experimental genetic analysis. Only approximately two-thirds of genes have an assigned biochemical function and a small percentage of these genes are associated with a phenotype (Bochner, 2003). This "phenotype gap," first described in mouse research (Brown and Peters, 1996), is a major challenge in biological understanding of plant processes and translational genomics. This gap must be filled by developing a coordinated effort to standardize phenotyping protocols across personnel, seasons, regions, and institutions and apply uniform phenotypic nomenclature to facilitate database searches, comparisons, and extrapolations.

Standardized phenotypic protocols are the prerequisite for the production of a so-called "phenomic map" in which every identified gene is defined by at least one phenotype. Such a system of comparative phenomics would facilitate the progression of knowledge throughout model biological systems in plants and from other well-studied organisms. As research projects become more nationally and internationally integrated, the need for standardized data collection and documentation has grown. We highlight several projects and germplasm centers that have recognized the need for developing standardized phenotyping protocols and the associated nomenclature, annotation, and databases for enabling comparative phenomics.

This workshop on standardized phenotyping and the advantages it provides for horticultural crops was held at the 2009 American Society for Horticultural Science Annual Conference in St. Louis, MO, and was organized by the Genetics and Germplasm Working Group. The objectives of the workshop were to 1) describe phenotyping efforts in diverse horticultural disciplines; 2) relate advantages for using standardized phenotyping for broad applicability in research and breeding programs; 3) emphasize the importance of standardization for genomics applications; and 4) give an update on standardized ontology for horticultural crops. Each of the five presentations was recorded and has been made available at http://www.ashs.org/db/horttalks/ listing2.lasso.

The five presentations include examples, advantages, constraints, and recommendations of/for the use standardized phenotyping in horticulture. The first speaker, Joseph Postman, related how "Standardized plant disease evaluations will enhance resistance gene discovery." He emphasized the value of using standardized numerical ratings and reference cultivars to record resistant and tolerant individuals. Jay Scott in "Phenotyping of tomato for SolCAP and onward into the void" and Jim Luby (for Cameron Peace and coworkers) in "Standardized phenotyping of apple texture for improved breeding and research efficiency" spoke of ongoing funded research programs in Solanaceae and Rosaceae, respectively, that have sought to use standardized recording methods across laboratories and locations to document germplasm performance. David Rudell presented "Standardized postharvest quality and biochemical phenotyping for precise population comparison" and described the value of standardization when documenting postharvest quality attributes in fruit in a quantitative manner. Gayle Volk concluded the workshop with a presentation "Advantages for the use of standardized phenotyping in the National Plant Germplasm System" that stressed the advantages and benefits for programs that overcome the difficulties involved in standardization, including examples from both the mammalian and plant systems. Postman, Scott, Rudell, and Volk have prepared manuscripts to document their American Society for Horticultural Science Workshop presentations.

\section{Literature Cited}

Bochner, B.R. 2003. New technologies to assess genotype-phenotype relationships. Nat. Rev. Genet. 4:309-314.

Brown, S.D. and J. Peters. 1996. Combining mutagenesis and genomics in the mouseClosing the phenotype gap. Trends Genet. 12: 433-435.
Received for publication 8 Mar. 2010. Accepted for publication 4 Apr. 2010.

${ }^{1}$ To whom reprint requests should be addressed; e-mail Nahla.bassil@ars.usda.gov. 